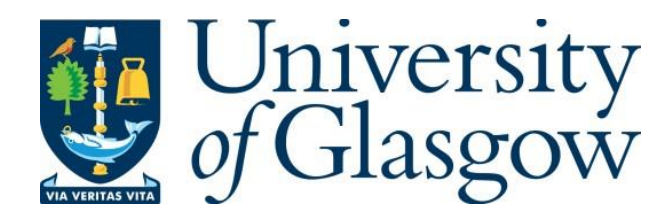

Bonfante, A., Giordano, L. G., López-Pérez, D., Garcia-Rodriguez, A., Geraci, G., Baracca, P., Butt, M. M., Dzaferagic, M. and Marchetti, N. (2019) Performance of Massive MIMO Self-Backhauling for Ultra-Dense Small Cell Deployments. In: IEEE GLOBECOM 2018, Abu Dhabi, United Arab Emirates, 09-13 Dec 2018, ISBN 9781538647271 .

There may be differences between this version and the published version. You are advised to consult the publisher's version if you wish to cite from it.

http://eprints.gla.ac.uk/166355/

Deposited on: 9 August 2018

Enlighten - Research publications by members of the University of Glasgow http://eprints.gla.ac.uk 


\title{
Performance of Massive MIMO Self-Backhauling for Ultra-Dense Small Cell Deployments
}

\author{
Andrea Bonfante* ${ }^{* \dagger}$ Lorenzo Galati Giordano*, David López-Pérez*, Adrian Garcia-Rodriguez*, Giovanni Geraci*, \\ Paolo Baracca ${ }^{\S}$, M. Majid Butt ${ }^{\ddagger}$, Merim Dzaferagic ${ }^{\dagger}$, and Nicola Marchetti ${ }^{\dagger}$ \\ ${ }^{*}$ Nokia Bell Labs Ireland and ${ }^{\S}$ Nokia Bell Labs Germany \\ ${ }^{\dagger}$ CONNECT Centre, Trinity College Dublin, Ireland \\ ¥University of Glasgow, United Kingdom
}

\begin{abstract}
A key aspect of the fifth-generation wireless communication network will be the integration of different services and technologies to provide seamless connectivity. In this paper, we consider using massive multiple-input multiple-output (mMIMO) to provide backhaul links to a dense deployment of self-backhauling (s-BH) small cells (SCs) that provide cellular access within the same spectrum resources of the backhaul. Through a comprehensive system-level simulation study, we evaluate the interplay between access and backhaul and the resulting end-to-end user rates. Moreover, we analyze the impact of different SCs deployment strategies, while varying the time resource allocation between radio access and backhaul links. We finally compare the above mMIMO-based s-BH approach to a mMIMO direct access (DA) architecture accounting for the effects of pilot reuse schemes, together with their associated overhead and contamination mitigation effects. The results show that dense SCs deployments supported by mMIMO s-BH provide significant rate improvements for cell-edge users (UEs) in ultradense deployments with respect to mMIMO DA, while the latter outperforms mMIMO s-BH from the median UEs' standpoint.

Index Terms-Integrated access and backhaul, heterogeneous network, massive MIMO-based backhaul, network capacity.
\end{abstract}

\section{INTRODUCTION}

Fifth-generation $(5 \mathrm{G})$ wireless communication systems are expected to support a 1000x increase in capacity compared to existing networks [1]. Meeting this gargantuan target will require mobile network operators (MNOs) to leverage new technologies such as massive multiple-input multiple-output (mMIMO), and deploy a large number of additional small cell base stations [2], [3]. Wireless self-backhauling (s-BH), achieved through the tight integration of these two complementary means, lures MNOs with the potential of achieving the desired capacity boost at a contained investment [4]. Indeed, exploiting the large number of spatial degrees-offreedom available with mMIMO to provide sub- $6 \mathrm{GHz}$ inband wireless backhauling to small cells (SCs) offers multiple advantages to MNOs: avoiding deployment of an expensive wired backhaul infrastructure, availing of more flexibility in

A. Bonfante was funded by the Irish Research Council and Nokia Ireland Ltd under the grant EPSPG/2016/106. This publication has emanated from research supported in part by a grant from Science Foundation Ireland (SFI) and is co-funded under the European Regional Development Fund under Grant Number 13/RC/2077. the deployment of SCs, and not having to purchase additional licensed spectrum as in the case of out-of-band wireless backhauling [5].

The integration of radio access and backhauling - advocated in s-BH solutions - has been addressed by the Third Generation Partnership Project (3GPP) with a list of requirements detailed in [6]. At the same time, various research efforts have tackled the problem of resource allocation and management in s-BH networks in the time and frequency domains [7], [8]. Finally, the combination of mMIMO spatial multiplexing and s-BH has been considered in [9]-[11] and also in [12]-[14], albeit for full-duplex scenarios.

In this paper, we analyze the end-to-end user equipment (UE) performance of mMIMO s-BH networks. In particular, we consider a realistic multi-cell setup where mMIMO base stations (mMIMO-BSs) provide sub- $6 \mathrm{GHz}$ backhauling to a plurality of half-duplex SCs overlaying the macro cellular area. We evaluate the UE data rates achieved through s-BH in two ultra-dense deployment scenarios, namely a random deployment - where SCs are uniformly distributed over a geographical area -, and an ad-hoc deployment - where SCs are purposely positioned close to UEs to achieve lineof-sight (LOS) access links. In these half-duplex systems, a s-BH approach entails sharing time-and-frequency resources between radio access and backhaul links. To the best of the authors knowledge, in this paper, we also compare for the first time the performance of the mMIMO s-BH approach and that of a direct access (DA) approach, where mMIMO-BSs are solely dedicated to serving UEs in the absence of SCs [15]. Our study provides a number of key takeaways:

- Adding more randomly deployed SCs - where SCs are randomly placed - provides limited gains for the end-toend UE rates. The UEs benefit from more radio resources allocated and from the SCs proximity, given by the higher probability of the UEs to be close to - and in LOS with - the serving SCs. However, the UEs are affected by a significantly higher inter-cell interference because they see a growing number of interfering links in LOS conditions. Overall, the detrimental impact of interference outweighs the combination of the gains provided by more 


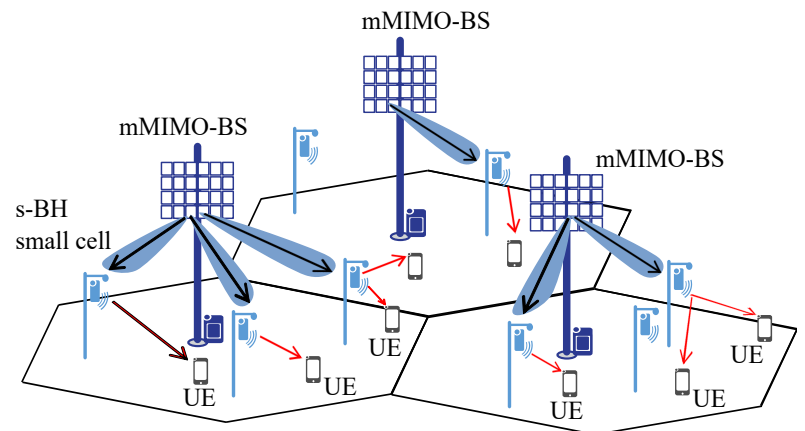

(a) Random deployment

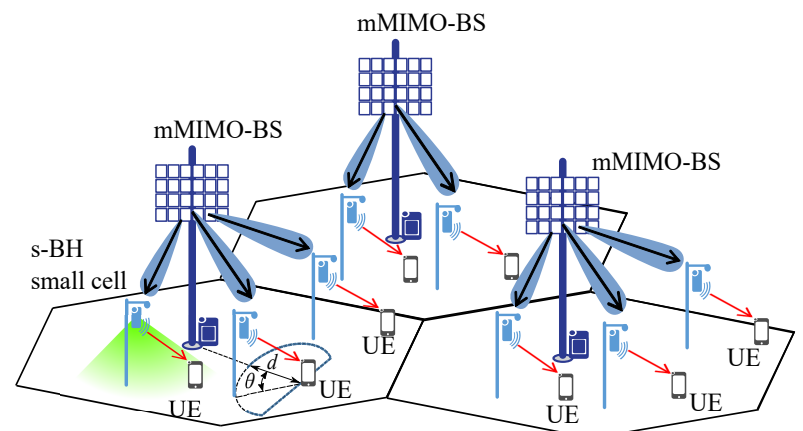

(b) Ad-hoc deployment

Fig. 1: Examples of two different SCs deployments considered in the paper.

radio resources and proximity, preventing the UEs to take the advantages of the SCs dense deployment.

- Adding ad-hoc deployed SCs - where SCs are placed in proximity to UEs - provides higher data rates, thanks to a high signal-to-interference-plus-noise ratio (SINR) on the access link, given by the higher proximity gains with respect to the random deployment.

- Partitioning resources between wireless access and backhaul links is of paramount importance. Indeed, the endto-end performance is sensitive to said partition, and optimal rates can only be achieved through a carefully designed tradeoff.

- Unlike mMIMO s-BH - where mMIMO-to-SC links are static, and thus channel acquisition is facilitated - mMIMO DA suffers more from pilot overhead and contamination. Indeed, when compared to mMIMO DA solutions with pilot reuse 3 and reuse 1, ultra-dense SCs deployments supported by mMIMO s-BH provide rate improvements for cell-edge UEs that amount to $30 \%$ and a tenfold gain, respectively. On the other hand, mMIMO DA outperforms s-BH from the median UEs' standpoint.

Notation: Capital and lower-case bold letters denote matrices and vectors, respectively, while $[\cdot]^{*},[\cdot]^{\mathrm{T}}$ and $[\cdot]^{\mathrm{H}}$ denote conjugate, transpose, and conjugate transpose, respectively.

\section{SySTEM MOdEL}

As shown in Fig. 1, we focus on the study of the downlink (DL) performance for a two-tier heterogeneous network formed by mMIMO-BSs overlaying a layer of self-backhauled SCs. The mMIMO-BSs are connected to the core network through a high-capacity wired connection, while all SCs receive backhaul traffic through mMIMO-BSs and function as access points for UEs. We consider a self-backhaul configuration, where mMIMO-BSs are solely dedicated for the backhaul, while SCs are solely dedicated for the access. For comparison purposes, we also consider the conventional DA approach where each mMIMO-BS directly serves the UEs.

\section{A. Macro cell and user topologies}

We denote by $\mathcal{I}$ the set of mMIMO-BSs placed in a uniform hexagonal grid with three sectors per site. Each mMIMO-BS $i$, is equipped with a large number of antennas $M$, and serves
$L_{i}$ single-antenna SCs. Furthermore, we denote by $K_{i}$ the number of UEs randomly and uniformly distributed over the sector's area, and let $k$ denotes single-antenna UE. We assume that each UE is connected with the SC (in the s-BH approach) or with the mMIMO-BS (in the DA approach) that provides the largest reference signal received power (RSRP) [16].

\section{B. Small cell topologies}

We denote by $\mathcal{L}_{i}$ the set of SCs deployed per sector and connected to the $i$-th mMIMO-BS that provides the largest RSRP. Each SC connects $K_{l}$ UEs. Two different SCs deployments are presented in the following:

(a) Random deployment: Self-backhauled SCs are randomly and uniformly distributed over the mMIMO-BS geographical area as shown in Fig. 1a. This scenario is used as a baseline and follows the set of parameters specified by the 3GPP in [16] to evaluate the relay scenario.

(b) Ad-hoc deployment: Self-backhauled SCs are positioned targeting nearby UE locations. This scenario is used as an example of ultra-dense network deployment. We assume the possibility to realize this target of network deployment, for example by means of drone-BSs, where the drone-BSs can reposition following the locations of UEs [17]. ${ }^{1}$ As shown in Fig. 1b, we model this scenario by considering SCs deployed within a 2-D (two-dimensional) distance $d$ of the UEs, and an angle $\theta$ measured from the straight segment that links UEs and their closest mMIMO-BS. $\theta$ is chosen uniformly at random from $-\pi / 2$ and $\pi / 2$. It is worth noting that even when the 2-D distance $d=0$, UEs and SCs are still separated in space because the antennas are positioned at different heights. More precisely, they are assumed located at 1.5 meters and 5 meters above the ground, for the UEs and the SCs, respectively [16].

With a dense deployment of SCs, the UE SINRs are severely affected by the strong inter-cell interference among

\footnotetext{
${ }^{1}$ Although mentioned, the drone-BSs use-case is not the focus of this paper and it is left for future investigation, since the height of the outdoor SC antenna is fixed to 5 meters, and we use the channel models adopted for the relay study [16].
} 
SCs. In addition, to limit the effect of the inter-cell interference, with the ad-hoc deployment, we propose to replace at the SC the Patch antenna with a more directive Yagi antenna pointing downwards to the ground (as shown by the green radiation cone in Fig. 1b), and therefore only illuminating the closest UEs: details about this modeling can be found in Table I.

\section{Frame structure}

As shown in Fig. 2a, we consider the time-slot $T$ as a single scheduling unit in the time domain, and we partition the access and backhauling resources through the parameter $\alpha \in$ $[0,1]$. Therefore, $\alpha$ time-slots are allocated to the backhaul links, while $1-\alpha$ time-slots are allocated to the access links. In the frequency domain, we divide the system bandwidth $B$ into $Q_{t}$ resource blocks ( $\mathrm{RBs}$ ), and we allocate all the $\mathrm{RBs}$ to the backhaul links or the access links. We make the following assumptions in considering the partition of backhaul and access time-slots among the SCs and UEs:

- During the backhaul time-slots, all the associated SCs are served by the mMIMO-BS $i$, and we use the same value of $\alpha$ for all the SCs. In this approach, the mMIMO-BSs precode the backhaul signals towards the single-antenna SCs, which are spatially multiplexed in the same timefrequency resources by allocating all the $\mathrm{RBs}$ in $T$ to each SC.

- During the access time-slots, the SCs schedule their connected UEs by using a Round Robin (RR) mechanism as frequency domain scheduler. This approach entails that each SC equally shares the system bandwidth $B$ with its UEs.

In the Fig. 2b, it is shown the frame structure used for the DA setup, where all the time-slots are allocated to the access links. In each time slot, the mMIMO BSs precode the access signals, and the UEs are spatially multiplexed on the entire system bandwidth. Figs. $2 a$ and $2 b$ also show the fraction $\tau$ of the time-slots dedicated for the transmission of the pilot sequences, used to estimate the massive MIMO channel. Details about the channel training procedure will be discussed in Section III.

\section{Channel model}

We define as $\mathbf{h}_{i l}=\left[h_{i l 1}, \ldots, h_{i l M}\right]^{\mathrm{T}} \in \mathbb{C}^{M}$ the propagation channel between the $l$-th single-antenna receiver (SC in the s-BH architecture and UE in the mMIMO DA) and the $M$ antennas of the $i$-th mMIMO-BS. The composite channel matrix between the $i$-th mMIMO-BS and the devices in the $i^{\prime}$-th cell is represented by $\mathbf{H}_{i, i^{\prime}}=\left[\mathbf{h}_{i 1} \cdots \mathbf{h}_{i L_{i^{\prime}}}\right] \in \mathbb{C}^{M \times L_{i^{\prime}}}$. Since all the RBs are assigned to each $\mathrm{SC}$, we removed the $\mathrm{RB}$ index $q$ from the massive MIMO channel notation.

Furthermore, we define as $g_{l k q} \in \mathbb{C}$ the single-input singleoutput (SISO) channel between the $l$-th SC and the $k$-th UE in the $q$-th RB. Each channel coefficient $h_{i l m}=\sqrt{\beta_{i l}} \tilde{h}_{i l m}$, and $g_{l k q}=\sqrt{\beta_{l k}} \tilde{g}_{l k q}$ accounts for both the effects of a large scale fading and a small scale fading components:

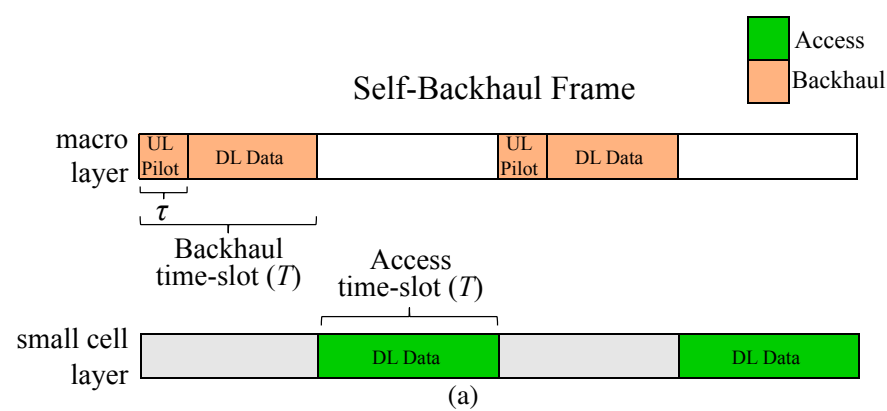

(a)

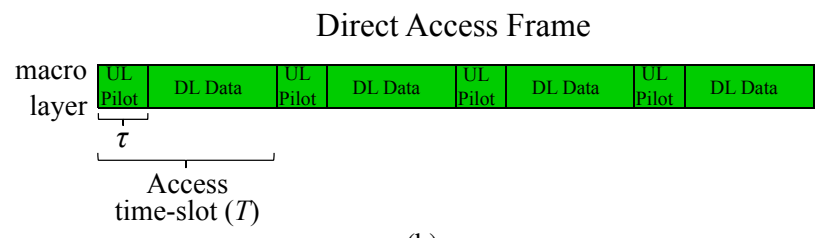

(b)

Fig. 2: DL frame structure for mMIMO s-BH with $\alpha=0.5$ (Fig. 2a) and for mMIMO DA (Fig. 2b).

- The large fading components $\beta_{i l}, \beta_{l k} \in \mathbb{R}^{+}$have been modeled by using a combined LOS/Non-LOS (NLOS) path loss model, which accounts for the shadowing effect, set to be log-normal distributed with different standard deviations [16]. Because of its slow-varying characteristic, it does not change rapidly with time, and it can be assumed constant over the observation time-scale of the network.

- The small scale fading components $\tilde{h}_{i l m}, \tilde{g}_{l k q} \in \mathbb{C}$, which results from multi-path, have been modeled as a Rician fast-fading, which rapidly changes over time and frequency. For the LOS channels, we characterize the Rician $K$ factor with the model: $K[d B]=13-0.03 r$ in $\mathrm{dB}$, where $r$ is the distance between transmitter and receiver in meters [18].

Throughout the paper, we assume a composite fading (i.e. large scale fading and small scale fading together) for the SC-UE and the mMIMO-BS-UE links (in the DA approach), which changes between successive time-slots and between different RBs. Moreover, because of the static position of the SCs, we consider that the backhaul channel SC-mMIMO-BS remains constant for a period $T_{B H} \gg T$.

\section{END-TO-END UE RATES}

In this section, we provide the detailed description of the operations required for the DL transmission in the mMIMO s$\mathrm{BH}$ approach and in the mMIMO DA approach. We describe the channel training procedure, the mMIMO DL backhaul transmission, and the DL access transmission, which is treated separately below for both the s-BH and the mMIMO DA setups.

\section{A. Massive MIMO channel training}

To calculate the DL precoder, we consider that the channel is estimated through uplink (UL) pilots, assuming UL/DL channel reciprocity [2]. We also assume that the SCs or UEs 
associated to the same mMIMO-BS have orthogonal pilot sequences, and define the pilot code-book with the matrix $\boldsymbol{\Phi}_{i}=\left[\boldsymbol{\phi}_{i 1} \cdots \phi_{i L_{i}}\right]^{\mathrm{T}} \in \mathbb{C}^{L_{i} \times S}$, which satisfies $\boldsymbol{\Phi}_{i} \boldsymbol{\Phi}_{i}^{\mathrm{H}}=\mathbf{I}_{L_{i}}$. Here, the $l$-th sequence is given by $\phi_{i l}=\left[\phi_{i l 1}, \ldots, \phi_{i l S}\right]^{\mathrm{T}} \in$ $\mathbb{C}^{S}$, and $S$ denotes the pilot code-book length. Note that $L_{i} \leq S$, i.e., the maximum number of SCs or UEs served by the mMIMO-BSs in a time-slot is limited by the number of orthogonal pilot sequences. The matrix $\mathbf{Y}_{i} \in \mathbb{C}^{M \times S}$ of pilot sequences received at the $i$-th mMIMO-BS can be expressed as [19]

$$
\mathbf{Y}_{i}=\sqrt{P_{i l}^{\mathrm{ul}}} \sum_{i^{\prime} \in \mathcal{I}} \mathbf{H}_{i, i^{\prime}} \boldsymbol{\Phi}_{i^{\prime}}+\mathbf{N}_{i}
$$

where $P_{i l}^{\mathrm{ul}}$ is the power used by the $l$-th device located in the $i$-th sector for UL pilot transmission, and $\mathbf{N}_{i} \in \mathbb{C}^{M \times S}$ represents an additive noise, and is modeled with independent and identically distributed complex Gaussian random variable.

Let $\mathbf{H}_{i}$ denote the channel between the $i$-th mMIMOBS and the UEs located in the same sector. During the UL training phase, the mMIMO-BS obtains an estimate of $\mathbf{H}_{i}$ by correlating the received signal with a known pilot matrix $\boldsymbol{\Phi}_{i}$. Let us define $\mathcal{P} \subseteq \mathcal{I}$ as the subset of sectors, whose UEs share identical pilot sequences with the UEs served by the $i$-th mMIMO-BS. The resulting estimated channel can be expressed as

$$
\widehat{\mathbf{H}}_{i}=\frac{1}{\sqrt{P_{i l}^{\mathrm{ul}}}} \mathbf{Y}_{i} \boldsymbol{\Phi}_{i}^{\mathrm{H}}=\mathbf{H}_{i}+\sum_{i^{\prime} \in \mathcal{P}} \mathbf{H}_{i, i^{\prime}}+\frac{1}{\sqrt{P_{i l}^{\mathrm{ul}}}} \mathbf{N}_{i} \boldsymbol{\Phi}_{i}^{\mathrm{H}} .
$$

The first, second and third terms on the right hand side of (2) represent the estimated channel, a residual pilot contamination component and the noise after the correlation, respectively. The use of the same set of orthogonal pilot sequences among different sectors leads to the well-known pilot contamination problem, which can severely degrade the performance of mMIMO systems [2], [20]. In this paper, we assume that no pilot contamination occurs for the mMIMO s-BH system. Due to the longer coherence time of the static backhaul channel, $T_{B H}$, with respect to the system time-slot, $T$, mMIMO pilots do not need to be transmitted in every time-slot dedicated to backhauling, thus allowing higher reuse factors with fully orthogonality over the entire network. In contrast, for mMIMO DA this assumption does not hold and, in this paper, we consider that a maximum of 16 orthogonal pilot sequences can be multiplexed in a single orthogonal frequency division multiplexing (OFDM) symbol [20]. In both mMIMO s-BH and mMIMO DA, the overhead associated to the UL training phase are considered and measured in terms of number of OFDM symbols $\tau$. Two pilot allocation schemes are here compared:

- Pilot reuse 1 scheme (R1): All $K_{i}$ UEs per sector are trained in $\tau=1$ OFDM symbol.

- Pilot reuse 3 scheme (R3): The sectors of the same site use orthogonal pilot sequences. This scheme avoids pilot contamination from co-sited sectors, but requires $\tau=$ 3 OFDM symbols, resulting in a higher pilot overhead when compared to the R1 scheme.

\section{B. Massive MIMO s-BH DL transmission}

The $i$-th mMIMO-BS uses the precoding matrix $\mathbf{W}_{i}=$ $\left[\mathbf{w}_{i 1} \cdots \mathbf{w}_{i L_{i}}\right] \in \mathbb{C}^{M \times L_{i}}$ to serve its connected UEs during the DL data transmission phase. In this paper, we consider that $\mathbf{W}_{i}$ is computed based on the zero-forcing (ZF) criterion as

$$
\mathbf{W}_{i}=\mathbf{D}_{i}^{\frac{1}{2}} \widehat{\mathbf{H}}_{i}\left(\widehat{\mathbf{H}}_{i}^{\mathrm{H}} \widehat{\mathbf{H}}_{i}\right)^{-1}
$$

Here, the diagonal matrix $\mathbf{D}_{i}=\operatorname{diag}\left(\rho_{i 1}, \rho_{i 2}, \ldots, \rho_{i L_{i}}\right)$ is chosen to equally distribute the total DL power $P_{i}^{\mathrm{dl}}$ among the $L_{i}$ receivers. In the previous expression, $\rho_{i l}$ represents the power allocated to the $l$-th receiver located in the $i$-th sector, and $\operatorname{Tr}\left\{\mathbf{D}_{i}\right\}=P_{i}^{\mathrm{dl}}$, where $\operatorname{Tr}\left\{\mathbf{D}_{i}\right\}$ is the trace of matrix $\mathbf{D}_{i}$.

The SINR of the $l$-th DL stream transmitted by the $i$-th mMIMO-BS can be expressed as

$$
\operatorname{SINR}_{i l}=\frac{\rho_{i l}\left|\mathbf{h}_{i l}^{\mathrm{H}} \mathbf{w}_{i l}\right|^{2}}{\sum_{\substack{j \in \mathcal{L}_{i} \\ j \neq l}} \rho_{i j}\left|\mathbf{h}_{i l}^{\mathrm{H}} \mathbf{w}_{i j}\right|^{2}+\sum_{\substack{i^{\prime} \in \mathcal{I} \\ i^{\prime} \neq i}} \sum_{j \in \mathcal{L}_{i^{\prime}}} \rho_{i^{\prime} j}\left|\mathbf{h}_{i^{\prime} l}^{\mathrm{H}} \mathbf{w}_{i^{\prime} j}\right|^{2}+\sigma_{n}^{2}} .
$$

The numerator of (4) contains the power of unit-variance signal intended for the $l$-th receiver, while the denominator includes the co-channel interference from the serving $i$-th mMIMO-BS, the inter-cell interference from other mMIMOBSs, and the power of the thermal noise at the receiver $\sigma_{n}^{2}$.

The corresponding DL backhauling rate at the $l$-th SC receiver can therefore be expressed as

$$
R_{i l}^{\mathrm{BH}}=\left(1-\frac{\tau}{T}\right) B \log _{2}\left(1+\mathrm{SINR}_{i l}\right) .
$$

\section{Small cell DL transmission}

We recall from the channel model that $g_{l k q}$ denotes the SISO channel between the $l$-th SC and the $k$-th UE corresponding to the $q$-th RB. The SINR of the $k$-th UE served by the $l$-th $\mathrm{SC}$ in $\mathrm{RB} q$ can be expressed as

$$
\operatorname{SINR}_{l k q}=\frac{P_{l}^{\mathrm{dl}}\left|g_{l k q}\right|^{2}}{\sum_{i \in \mathcal{I}} \sum_{\substack{l^{\prime} \in \mathcal{L}_{i} \\ l^{\prime} \neq l}} P_{l^{\prime}}^{\mathrm{d} 1}\left|g_{l^{\prime} k q}\right|^{2}+\sigma_{n_{2}}^{2}},
$$

where $P_{l}^{\mathrm{dl}}$ and $P_{l^{\prime}}^{\mathrm{dl}}$ are the transmit powers on the RB of the $l$-th and $l^{\prime}$-th SCs, respectively, and $\sigma_{n_{2}}^{2}$ denotes the thermal noise power at the UE receiver. The DL access rate for UE $k$ served by SC $l$ can be therefore expressed as

$$
R_{l k}^{\mathrm{AC}}=\frac{B}{Q_{t}} \sum_{q=1}^{Q_{t}} x_{q}^{k} \log _{2}\left(1+\mathrm{SINR}_{l k q}\right),
$$

where $x_{q}^{k}=1$ if the $q$-th resource block is assigned to the $k$-th user, and $x_{q}^{k}=0$ otherwise. The aggregated DL access rate provided by the $l$-th $\mathrm{SC}$ is $R_{l}^{\mathrm{AC}}=\sum_{k=1}^{K_{l}} R_{l k}^{\mathrm{AC}}$. The actual aggregated DL access rate provided by the $l$-th SC depends on the backhaul DL rate, which entails that $R_{l}^{\mathrm{AC}} \leq R_{i l}^{\mathrm{BH}}, \forall l \in$ $\mathcal{L}_{i}$, and $\forall i \in \mathcal{I}$. In this paper, we assume that the backhaul capacity is equally divided between the $K_{l}$ UEs served by the 
$l$-th SC. ${ }^{2}$ Therefore, the resulting end-to-end access rate for the $k$-th UE can be expressed as

$$
R_{i l k}=\min \left(\alpha \frac{R_{i l}^{\mathrm{BH}}}{K_{l}},(1-\alpha) R_{l k}^{\mathrm{AC}}\right),
$$

where $\alpha$, as indicated before, represents the time-slots allocated to the backhaul links.

\section{Massive MIMO direct access transmission}

In contrast to s-BH setups, mMIMO systems providing DA dedicate all their time resources to DL data transmission. Therefore, the DL access rate of the $k$-th UE served by the $i$-th mMIMO-BS can be expressed as

$$
R_{i k}^{\mathrm{AC}}=\left(1-\frac{\tau}{T}\right) B \log _{2}\left(1+\mathrm{SINR}_{i k}\right),
$$

where the estimated channel matrix $\widehat{\mathbf{H}}_{i}=\left[\widehat{\mathbf{h}}_{i 1} \cdots \widehat{\mathbf{h}}_{i K_{i}}\right] \in$ $\mathbb{C}^{M \times K_{i}}$ between the $i$-th mMIMO-BS and its connected UEs is plugged into (3), to subsequently derive (4) and (9).

\section{Numerical Results}

To realistically evaluate the mMIMO s-BH network performance, in this paper, we adopt the methodology described by 3 GPP in [16] for heterogeneous network. We perform system level simulations accounting for all signal and interfering radio links between each SC and the UEs, as well as between each mMIMO-BS and all SCs. We collect statistics for different network realizations, each with independent deployments of UEs and SCs. Subsequently, we measure the performance in terms of cumulative distribution function (CDF) of the end-to-end UE rate (8). To compare s-BH against DA, we also simulate the links between mMIMO-BSs and UEs, and compute the resultant rates (9). Table I contains the relevant parameters used to conduct the simulation campaign.

\section{A. Small cell random and ad-hoc deployments with mMIMO $s-B H$}

In Fig. 3, we assume $\alpha=0.5$, and analyze the results for the two SC topologies described in Sec. II-B, namely the ad-hoc and random SC deployments. In both cases, $K_{i}=16 \mathrm{UEs}$ are deployed per sector, and scheduled in access time-slots by their serving SCs. We evaluate the impact of densification by considering $L_{i}=\{4,8,16\}$ SCs per sector for the case of random SC deployments. In the ad-hoc deployment, we consider $L_{i}=16 \mathrm{SCs}$ per sector, and different values of the 2-D UE-to-SC distance $d$.

The results of Fig. 3 illustrate that the improvements attained by adding more SCs in the random deployment scenario are limited. This occurs because when densifying the network the carrier signal benefits from having SCs that are more likely in close vicinity with the served UE. Moreover, the UEs

\footnotetext{
${ }^{2}$ The assumption of equally distributed backhaul capacity might become a drawback for the end-to-end rates when UEs served by the same SC have significant differences between the rates of the access links, and in this case, the partition of the backhaul resources among the UEs could be designed proportionally to their access rates. This access-based partition of the backhaul resources among the UEs is not the focus of this paper, and its study in the context of self-backhaul is left for future work.
}

\begin{tabular}{|c|c|}
\hline mMIMO-BSs & Description \\
\hline Cellular layout & $\begin{array}{l}\text { Wrap around hexagonal, } 19 \text { sites, } 3 \text { sec- } \\
\text { tors/site }\end{array}$ \\
\hline Deployment & Inter-site distance: $500 \mathrm{~m}$, height: $32 \mathrm{~m} \mathrm{[16]}$ \\
\hline Antenna array & $\begin{array}{l}\text { Uniform linear, spacing: } 0.5 \lambda \text {, Number of } \\
\text { antennas per array: } 64\end{array}$ \\
\hline Antenna pattern & $\begin{array}{l}70^{\circ} \mathrm{H} \times 10^{\circ} \mathrm{V} \text { beamwidths, } 14 \mathrm{dBi} \text { max., } \\
\text { downtilt: } 15^{\circ}[16]\end{array}$ \\
\hline Precoder & Zero-forcing \\
\hline Tx power/Noise figure & $46 \mathrm{dBm}, 5 \mathrm{dBm}[16]$ \\
\hline Self-BH SCs & Description \\
\hline Deployment & $\begin{array}{l}\text { Random: }\{4,8,16\} \text { SCs/sector, Ad-hoc: } 16 \\
\text { SCs/sector, height: } 5 \mathrm{~m}\end{array}$ \\
\hline Backhaul antenna pattern & $5 \mathrm{dBi}$ antenna gain, Omni [16] \\
\hline $\begin{array}{l}\text { Access antenna pattern - } \\
\text { Patch }\end{array}$ & $\begin{array}{l}80^{\circ} \mathrm{H} \times 80^{\circ} \mathrm{V} \text { beamwidths, } 5 \mathrm{dBi} \text { max., } \\
\text { downtilt: } 90^{\circ}\end{array}$ \\
\hline $\begin{array}{l}\text { Access antenna pattern - } \\
\text { Yagi }\end{array}$ & $\begin{array}{l}58^{\circ} \mathrm{H} \times 47^{\circ} \mathrm{V} \text { beamwidths, } 10 \mathrm{dBi} \text { max., } \\
\text { downtilt: } 90^{\circ}\end{array}$ \\
\hline Tx power/Noise figure & $30 \mathrm{dBm}, 5 \mathrm{~dB}[16]$ \\
\hline UEs & Description \\
\hline Deployment & $\begin{array}{l}\text { Random, } 16 \text { UEs/sector on average, all } \\
\text { served, height: } 1.5 \mathrm{~m}\end{array}$ \\
\hline Tx power/Noise figure & $23 \mathrm{dBm}, 9 \mathrm{~dB}[16]$ \\
\hline Channel & Description \\
\hline Scenario & Outdoor SCs, outdoor UEs \\
\hline Bandwidth/Time-slot & $\begin{array}{l}10 \mathrm{MHz} \text { at } 2 \mathrm{GHz}, Q_{t}=50 \mathrm{RBs}, T=1 \\
\text { msec. [16] }\end{array}$ \\
\hline $\begin{array}{l}\text { LOS probability, path } \\
\text { loss and shadowing }\end{array}$ & $\begin{array}{l}\text { mMIMO-BS to UE (based on } 3 \mathrm{GPP} \text { macro } \\
\text { to UE models), mMIMO-BS to SC (based } \\
\text { on } 3 \text { GPP macro to relay models), SC to UE } \\
\text { (based on } 3 \text { GPP relay to UE models) as per } \\
\text { [16] }\end{array}$ \\
\hline Fast fading & Rician, distance-dependent K-factor [18] \\
\hline Thermal noise & $-174 \mathrm{dBm} / \mathrm{Hz}$ power spectral density \\
\hline
\end{tabular}

TABLE I: Simulation parameters

benefit from more radio resources allocated. In fact, with more SCs, there are less UEs served per SC, and the SC can allocate more RBs per UE. However, adding more SCs increase the probability of having a larger number of interfering SCs with a LOS channel with respect to the UE. As a result, the power of the interference links grows faster than the carrier signal power due to NLOS to LOS transition of the interference links [21]. The gains provided by more radio resources and proximity are outweighed by the detrimental impact of interference, and from the curves shown in Fig. 3, we can see that the end-toend UE rates increase marginally when doubling the number of SCs deployed.

Regarding the ad-hoc deployment, the results of Fig. 3 demonstrate that decreasing $d$ leads to significant improvements in the UEs rates. This is because UEs benefit from a robust LOS component and reduced path loss, which increases signal power gains and consequently leads to larger SINRs. Fig. 3 also illustrates the performance of two antenna patterns (Patch and Yagi) mounted at the SC. It can be observed that SCs equipped with a more directive antenna for access (i.e. Yagi) provide higher performance than those equipped with the Patch antenna. The performance enhancement is caused by two complementary effects: $i$ ) the signal improvements provided by the larger antenna gain of the directive Yagi, and ii) the reduced interference created towards neighboring UEs served by other SCs. 


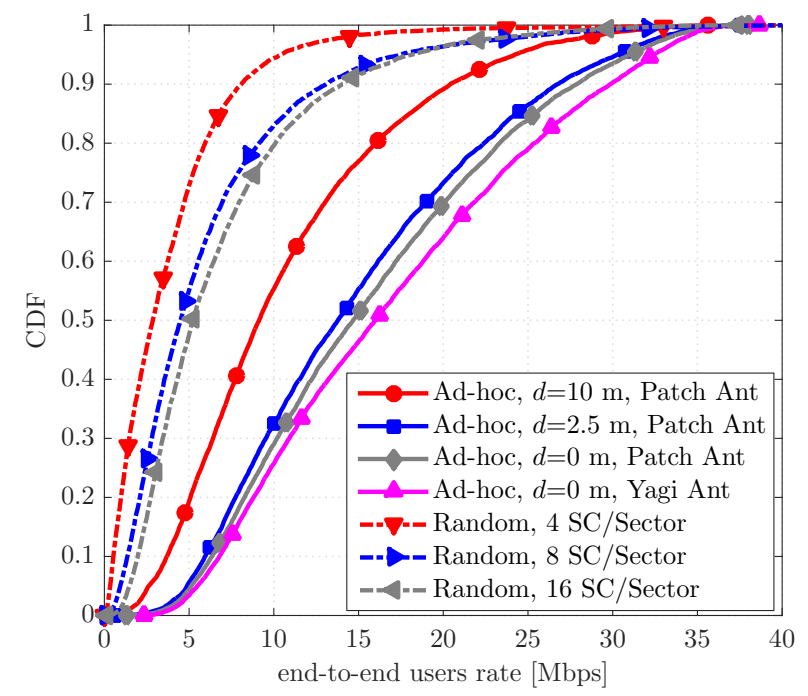

Fig. 3: CDF of end-to-end UE rate in: $(i)$ ad-hoc deployment of 16 SCs per sector with variable UE-to-SC distance $d$ and different antenna patterns (Patch and Yagi); (ii) random deployment of SCs.

\section{B. Impact of the resource allocation}

In Fig. 4, we vary $\alpha$ in the range $0 \leq \alpha \leq 1$ to analyze the behavior of UEs rate at the 5-th and 50-th percentiles of the CDF. The configurations $\alpha=0$ and $\alpha=1$ entail that all the time-slots are allocated to the access and the backhaul, respectively. Therefore, the UE rate for these two values is equal to 0 , since no resources are left for the other link. Fig. 4 brings the following insights:

- The s-BH links are generally the bottleneck of the two hop connection when the SCs are densely deployed near the UEs. In such a case, in order to take advantage of the very high SINR experienced by the UEs on the access link, most of the resources must be allocated to the backhaul. For instance, with $d=0$ and Yagi antennas at the SCs, $\alpha^{*}$, i.e., the value of $\alpha$ that maximizes the UE rate, is about 0.85 when looking at the 5 -th percentile curve.

- By comparing the results between Fig. 4a and Fig. 4b, it is important to notice that the optimal $\alpha$ changes from 0.85 to 0.75 . This deviation suggests that picking a nonoptimal $\alpha$ can lead to a significant reduction of the endto-end UE rates. In fact, assuming that the network uses $\alpha=0.85$, which is the optimal value for cell-edge UEs (5-th percentile of the CDF), the median UEs (50-th percentile of the CDF) can achieve an end-to-end rate of $19.5 \mathrm{Mbps}$, which represents a $16 \%$ reduction with respect to the maximum end-to-end rate achievable of 23.3 Mbps.

- In Figs. 4a and 4b, we show with dash lines the results of the mMIMO DA setup, which is considered as the baseline for the network performance. The results show that a properly designed s-BH system can improve the performance of the cell-edge UEs, but this is not the case for the median-UEs. A more detailed comparison is further developed in the next section.

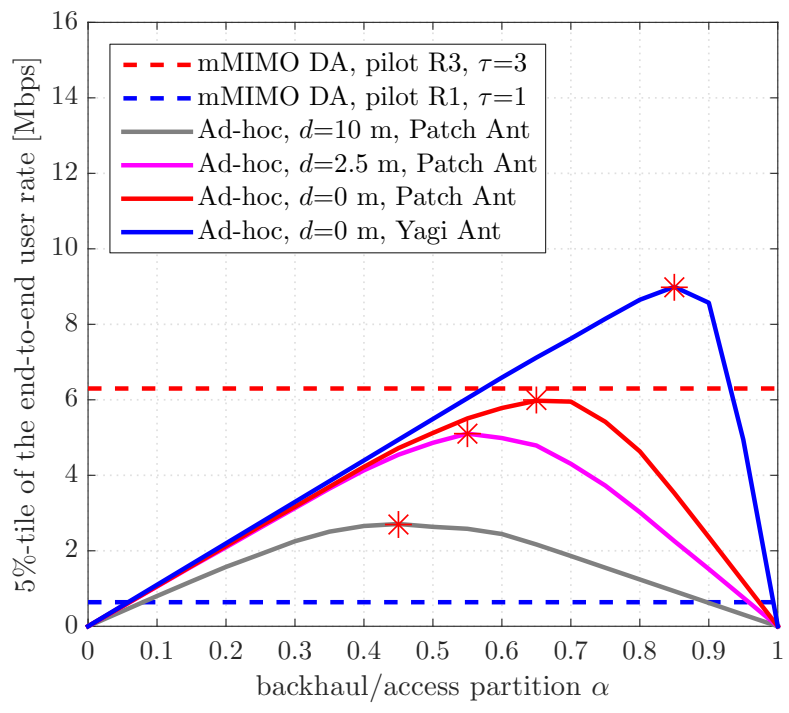

(a) 5 -th percentile

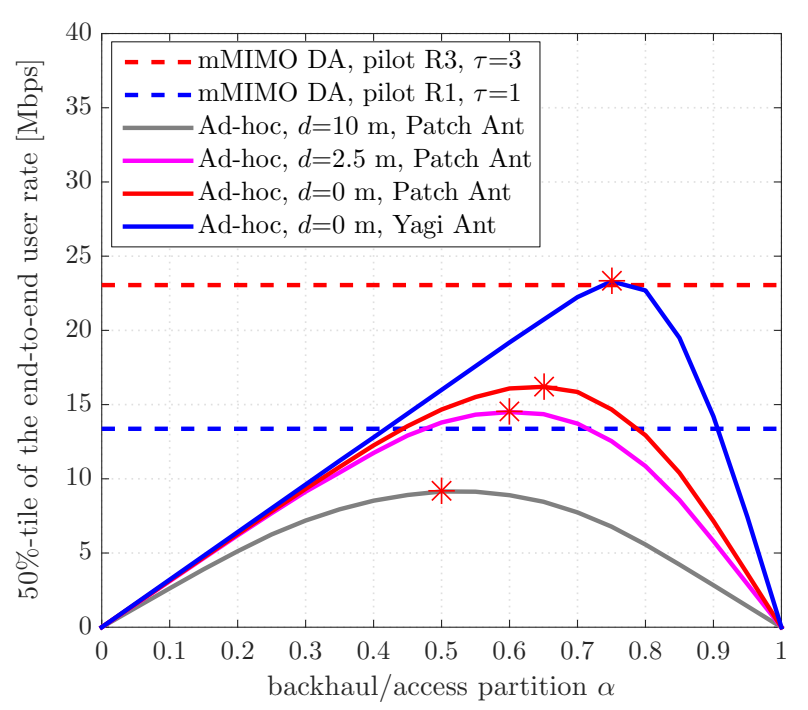

(b) 50-th percentile

Fig. 4: (a) 5-th, and (b) 50-th percentile of the UE rates as a function of the partition $\alpha$ between backhaul and access time-slots.

\section{Comparison between DA and s-BH systems}

In this section, we further compare the mMIMO s-BH and the mMIMO DA architectures to identify in which conditions the network results in a better performance in terms of UE rates. For the mMIMO DA architecture, $K_{i}=16$ UEs are trained and served per time-slot $T$. From Fig. 5, we identify two different regions:

- At the bottom of the CDF, i.e. below the 50-th percentile, the mMIMO s-BH architecture with $d=0$ and Yagi antennas provides better performance when compared to the mMIMO DA architecture with pilot reuse 1. This is because pilot contamination severely degrades the rate of UEs at the cell edge in the mMIMO DA setup with pilot reuse 1. In this region, s-BH architecture works better because: 1) access links benefit from the UE-to-SC proximity, which reduces the path loss and improve the 


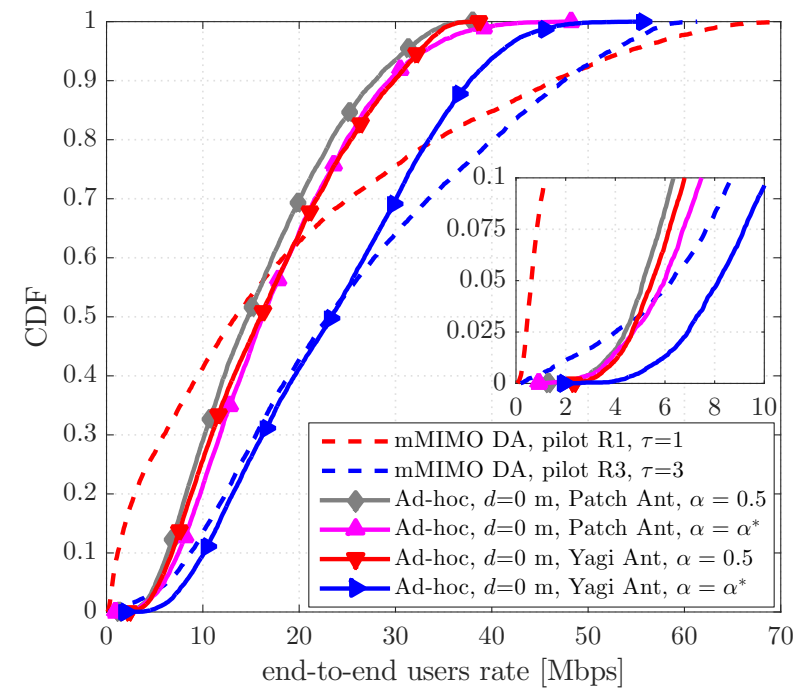

Fig. 5: Two types of curves are represented: (i) mMIMO DA with pilot reuse schemes 1 and 3; (ii) ad-hoc deployment of 16 SCs per sector for $\alpha=0.5$ and $\alpha=\alpha^{*}$, at which the 50-th percentile of the UE rate is maximized (as shown in Fig. 4b).

LOS propagation condition, and 2) backhaul links benefit from the absence of pilot contamination, and the higher height of the SC compared to the UE. The latter leads to an improved path-loss and LOS conditions with respect to those modelled for the macro to UE link [16]. The gain achieved by mMIMO s-BH decreases when mMIMO DA with pilot reuse 3 is considered, mainly because of the reduced pilot contamination effect in the latter. However, we still observe some considerable gain of about $30 \%$ with $d=0$ and Yagi antennas when looking at the 5-th percentile of the UE rate.

- At the top of the CDF, i.e. over the 50-th percentile, the mMIMO DA architecture exceeds the performance of s$\mathrm{BH}$ mMIMO. This is because, with s-BH, the end-to-end rates are conditioned by both inter-cell interference and limited backhaul capacity, which combined set a limit to the maximum rate of the two hop connection.

The results of Fig. 5 confirm that the deployment of s-BH architectures can be effective for serving UEs located at the cell edge and motivate the use of mMIMO DA for serving median UEs.

\section{CONClusion}

In this paper, we analyzed the performance of the mMIMO based s-BH architecture below $6 \mathrm{GHz}$ frequencies. We adopted a system-level simulation approach to investigate the UE rate performance for different SC deployments, and to analyze the effect of the variation of the backhaul/access partition. We showed that end-to-end performance greatly benefits from an ad-hoc SC deployment with one SC per UE, and studied the optimal backhaul/access partition, which maximizes the end-to-end rates, for the cell-edge UEs and for the median UEs. Additionally, we also study the effect of that antenna directivity adopted at the SCs, which leads to important gains in a dense deployment of SCs. Finally, we compared the s-BH architecture against a mMIMO DA baseline. By properly optimizing the SC deployment and antenna directivity, s-BH outperforms DA at the cell edge in ultra-dense deployments, in particular when pilot reuse 1 is used in the latter. On the other hand, DA outperforms s-BH when looking at the median UEs.

\section{REFERENCES}

[1] A. Gupta et al., "A survey of 5G network: Architecture and emerging technologies," IEEE Access, vol. 3, pp. 1206-1232, Jul 2015.

[2] F. Rusek et al., "Scaling up MIMO: Opportunities and challenges with very large arrays," IEEE Signal Processing Magazine, vol. 30, no. 1, pp. 40-60, Jan 2013.

[3] D. Lopez-Perez et al., "Towards $1 \mathrm{Gbps} / \mathrm{UE}$ in cellular systems: Understanding ultra-dense small cell deployments," IEEE Signal Processing Mag., vol. 17, no. 4, pp. 2078-2101, 4th Quart., 2015.

[4] U. Siddique et al., "Wireless backhauling of 5G small cells: challenges and solution approaches," IEEE Wireless Communications, vol. 22, no. 5, pp. 22-31, Oct 2015.

[5] N. Wang et al., "Backhauling 5G small cells: A radio resource management perspective," IEEE Wireless Communications, vol. 22, no. 5, pp. 41-49, Oct 2015.

[6] 3GPP 22.261, "Service requirements for the 5G system," Technical Specification (TS), Sep 2017.

[7] R. Gupta et al., "Resource allocation for self-backhauled networks with half-duplex small cells," in Proc. IEEE Int. Conf. Commun. (ICC), May 2017, pp. 198-204.

[8] T. M. Nguyen et al., "Resource allocation in two-tier wireless backhaul heterogeneous networks," IEEE Trans. Wireless Commun., vol. 15, no. 10, pp. 6690-6704, Oct 2016.

[9] P. Kela et al., "Flexible backhauling with massive MIMO for ultra-dense networks," IEEE Access, vol. 4, pp. 9625-9634, Dec 2016.

[10] H. H. Yang et al., "Energy-efficient design of MIMO heterogeneous networks with wireless backhaul," IEEE Trans. Wireless Commun., vol. 15 , no. 7 , pp. 4914-4927, July 2016.

[11] M. Feng et al., "Joint frame design, resource allocation and user association for massive mimo heterogeneous networks with wireless backhaul," IEEE Transactions on Wireless Communications, vol. 17, no. 3, pp. 1937-1950, March 2018.

[12] H. Tabassum et al., "Analysis of massive MIMO-enabled downlink wireless backhauling for full-duplex small cells," IEEE Trans. Commun., vol. 64, no. 6, pp. 2354-2369, June 2016.

[13] B. Li et al., "Small cell in-band wireless backhaul in massive MIMO systems: A cooperation of next-generation techniques," IEEE Trans. Wireless Commun., vol. 14, no. 12, pp. 7057-7069, Dec 2015.

[14] W. Lv et al., "Interference coordination in full-duplex HetNet with large-scale antenna arrays," in 2017 IEEE International Conference on Communications (ICC), May 2017, pp. 1-6.

[15] Y. G. Lim et al., "Performance analysis of massive MIMO for cellboundary users," IEEE Trans. Wireless Commun., vol. 14, no. 12, pp. 6827-6842, Dec 2015.

[16] 3GPP 36.814, "Further advancements for E-UTRA physical layer aspects," Technical Report (TR), Mar 2017.

[17] I. Bor-Yaliniz et al., "The new frontier in RAN heterogeneity: Multitier drone-cells," IEEE Communications Magazine, vol. 54, no. 11, pp. 48-55, November 2016.

[18] 3GPP 25.996, "Spatial channel model for Multiple Input Multiple Output (MIMO) simulations (Release 14)," Technical Report (TR), Mar 2017.

[19] X. Zhu et al., "Soft pilot reuse and multicell block diagonalization precoding for massive MIMO systems," IEEE Trans. Veh. Technol., vol. 65, no. 5, pp. 3285-3298, May 2016.

[20] L. G. Giordano et al., "Uplink sounding reference signal coordination to combat pilot contamination in 5G massive MIMO," in 2018 IEEE Wireless Communications and Networking Conference (WCNC), April 2018, pp. 1-6.

[21] M. Ding et al., "Performance impact of LoS and NLoS transmissions in dense cellular networks," IEEE Transactions on Wireless Communications, vol. 15, no. 3, pp. 2365-2380, March 2016. 